\title{
3 Bild und Bildkonstruktionen: Die Perspektive des Nuntius
}

Die Beschäftigung mit kulturellen Bildkonstruktionen ${ }^{1}$ gehörte bisher vor allem zum Bereich der Soziologie und vor allem der sogenannten cultural studies. Wie Müller-Funk in seiner Einführung zum Thema schreibt, gehört „die Beschäftigung mit der Figur des Fremden“ „seit mehreren Jahrzehnten zum unverzichtbaren Bestandteil gegenwärtiger kultureller, sozialer sowie politischer Diskurse und Debatten“. ${ }^{2}$ Es gehöre zur Entwicklung jedoch auch dazu, ,dass die Bedeutung des Fremden wie auch des Eigenen im Wandel begriffen sind“.”3 Man müsse hierbei zwischen mindestens drei einander überlappenden Alteritätsphänomenen unterscheiden und zwar: Andersheit (Zweiheit), Fremdheit (Unbekanntheit) und Ausländisch-Sein (Extraterritorialität). ${ }^{4}$

Bei den Nuntien im 16. und 17. Jahrhundert in Luzern spielen diese Elemente eine andere Rolle. Sie waren im Auftrag des religiösen Oberhauptes in der Schweiz, und somit fühlten sie sich den Gastgebern zumindest im kirchlichhierarchischen Sinne überlegen. Auch wenn die Schweizer den Nuntien zunächst „,befremdlich“ erscheinen sollten, hatten sie dennoch nicht mit „Unbekannten“ zu tun. Im Gegenteil, durch den täglichen Austausch und die Behandlung von konkreten Themen waren sie den Schweizern - zumindest hatten sie dieses Gefühl - sehr nahe. Einzig das Ausländisch-Sein kann hier zweifelsohne angebracht werden. Bereits die sprachlichen Unterschiede und die geographischen Begebenheiten ließen den Nuntien keinen Zweifel daran, dass sie in einem ,anderen“ Land waren. ${ }^{5}$

Wie Müller-Funk hervorhebt, hat die „Exterritorialität“, das „Ausländische“, eine „unverkennbar qualitative Bestimmung“, deshalb gehöre diese Phänomenenlage „zum Aspekt der Identität im engeren Sinne“. 6

Wenn wir die Komparatistik ${ }^{7}$ zu Rate ziehen, dann können wir feststellen, dass die Korrespondenz und die Instruktionen ein klares Bild der Schweiz und

1 Vgl. Wolfgang Müller-Funk (Hrsg.): Theorien des Fremden. Da die Literatur und Erforschung in diesem Bereich sehr breit und komplex ist, wird vor allem auf das von Müller-Funk herausgegebene Werk verwiesen und anhand dieser Publikation die Bild-Konstruktion erarbeitet.

2 Ebd., S. 15.

3 Ebd., S. 15.

4 Ebd., S. 22.

5 Vgl. Vgl. Christoph Wulf: Bilder des Menschen, S. 21: „Nach wie vor fällt es schwer zu begreifen, dass unsere Art, die Welt als Bild und in Bildern zu sehen, historisch und kulturell bedingt ist."

6 Ebd., S. 23.

7 Ebd., S. 191. Müller-Funk geht hierbei auf die Imagologie als Methode und Disziplin ein.

ə Open Access. (C) 2021 Mario Galgano, publiziert von De Gruyter. $(\leftrightarrow)$ Er Dieses Werk ist lizenziert unter der Creative Commons Attribution 4.0 Lizenz. Weitere Informationen finden Sie unter 
der Schweizer darstellen und zwar durchgehend gleichbleibend und fix. MüllerFunk betont, dass die „Stereotype, die von einer Kultur hinsichtlich einer anderen formuliert und festgehalten werden“, problematisch sein können - man denke an die negativen Vorurteile gegenüber Flüchtlingen oder im 20. Jahrhundert die Darstellung der Juden im Dritten Reich -, aber gleichzeitig könne es auch „wirksame Formen der interkulturellen Begegnung“ geben. ${ }^{8}$ Wer also „ein anderes Land und seine Menschen betrachtet, beurteilt und beschreibt, der betrachtet sie nicht nur aus seiner persönlichen Perspektive, sondern er vergleicht die anderen auch mit dem Land seiner Herkunft und den Eigenschaften seiner Landsleute".9

Somit waren die Nuntien einerseits „gefangen“ in der Vorstellung, die man in den Instruktionen vorfand, und andererseits war der kulturelle Hintergrund der Nuntien bei allen mehr oder weniger gleich. Dies förderte somit ein gleichbleibendes Bild. ${ }^{10}$

Die Nuntien in der Schweiz hatten bekanntlich auch keine direkte und zentrale Ansprechperson. Selbst die Tagsatzung, die üblicherweise in Baden durchgeführt wurde, war keine parlamentarische Versammlung oder Landsgemeinde-Treffen, sondern, wie Jucker schreibt, „ein lockeres Ad-hoc-Forum, welches durch die verschiedensten Faktoren wie Schriftlichkeitsgrad, Geschenkpolitik, aktuelle politische Koalitionen und Tagesgeschäfte geprägt war und bis ins 16 . Jahrhundert hinein nie eine, wie in der Literatur teilweise angenommen wurde, zentralbehördliche Funktion einnahm". ${ }^{11}$

Wie Jucker ebenfalls betont, seien Diplomatie und Verhandlungen „immer auch geprägt von einer Politik der Zeichen und von Visualisierungs- und Sichtbarkeitsstrategien"“. ${ }^{12}$ So spielten für die Nuntien in Luzern die Rituale, Kleidung und Devisen eine große Rolle, sowie die Umgangsformen, die sie aus einer katholisch-moralischen Perspektive betrachteten, die den Vorgaben des Konzils von Trient entsprechen mussten.

Die Diplomatie der Päpste und der römischen Kurie nimmt ihren Ursprung um die Mitte des 15. Jahrhunderts. Waren es ursprünglich vor allem Gesandtschaften bei den italienischen Kleinstaaten, so entwickelte sich im Laufe der

8 Vgl. ebd., S. 191.

9 Zit. nach: Dietrich Harth (Hrsg.): Fiktion des Fremden.

10 Vgl. Christoph Wulf, Bilder des Menschen, S. 89: „Das Imaginäre ist einer Karte vergleichbar, mit deren Hilfe wir die Welt in dem Bewusstsein lesen, dass sie uns als reale nicht zugänglich ist. Das Imaginäre ist performativ; es erzeugt Bilder und schafft Ordnung; zugleich ist es selbst das Ergebnis inkorporierter Bilder und Ordnungen. Seine Bilder vermitteln zwischen uns, der Welt und den anderen Menschen.“

11 Zit. nach: Michael Jucker: Ein einig Volk von Brüdern?, S. 38.

12 Vgl. ebd., S. 42. 
Zeit eine Entsendung außerhalb der italienischen Halbinsel. ${ }^{13}$ Die ständigen Nuntiaturen waren im 16. Jahrhundert nicht aus der Tradition entstanden, wie man meinen könnte. Es gab beispielsweise bereits Vertreter des Papstes am byzantinischen Hof, doch diese „Erfahrungen“ hatten keinen Einfluss auf die Errichtung der ständigen Nuntiaturen. ${ }^{14}$ Vielmehr ist es so, dass durch mehrere aufeinander folgende Nuntien eine ständige Nuntiatur gebildet wurde. Im Falle der Eidgenossenschaft war es insofern anders, als dass dies mit dem Druck Borromäus' noch gefördert wurde. Doch auch für die Eidgenossenschaft galt die Regel, dass die ständige Nuntiatur nach einer Abfolge von bereits präexistierenden Gesandtschaften als eine ständige Nuntiatur eingerichtet wurde. ${ }^{15}$

\subsection{Historische Entwicklung: Welche Ereignisse die Nuntien prägten}

Im 16. Jahrhundert entstanden päpstliche Nuntiaturen als ständige Einrichtungen des Heiligen Stuhls zunächst an den katholischen Königshöfen, so zum Beispiel in Wien und Graz, in Madrid, oder bei Kurfürsten. Als älteste Einrichtung dieser Art gilt die ständige Nuntiatur am Hof König Ferdinands I., 1529 in Wien eingerichtet.

Wie Surchat feststellte, „liefen die Uhren der damaligen Eidgenossenschaft anders“. ${ }^{16}$ Die Geschichtsbücher berichten heute weiterhin mehrheitlich, dass die damalige Schweiz „mehr oder weniger vom Krieg verschont geblieben“ sei. Dies habe „eine gewisse Lethargie“ mit sich gebracht, die durch die „politische und konfessionelle Uneinigkeit erzeugt“ worden sei, schreibt Surchat weiter; so habe es „den Anschein“ gegeben, „als lägen die Kantone abseits vom Weltgeschehen“. Schaut man sich die Korrespondenz der Nuntien genauer an, so fällt auf, dass diese Hypothese falsch ist. Denn die Eidgenossenschaft blieb alles andere als verschont von der Entwicklung in Europa und insbesondere von den Wirren im Deutschen Reich. Was die damaligen Großmächte Europas unternahmen, betraf auch die Schweiz, und dabei ging es nicht nur um „rein konfessionelle Auseinandersetzungen“, wie Surchat betont. ${ }^{17}$ So hatten die Staatsinteressen begonnen, den religiösen gegenüber „,an Vorrang zu gewinnen“. Und das

13 Vgl. Anton Pieper Zur Entstehungsgeschichte der ständigen Nuntiaturen, S. 1.

14 Vgl. ebd., S. 2.

15 Weiterführende Literatur zu dem Thema „Bild und Bildkonstruktionen“: Christoph Antweiler: Was ist den Menschen gemeinsam? Über Kultur und Kulturen. Darmstadt 2007.

16 Zit. nach: Pierre Louis Surchat: Die Nuntiatur von Ranuccio Scotti in Luzern 1630-1639, S. 24. Dieser gesamte Absatz bezieht sich auf diese Seite in Surchats Werk.

17 Vgl. ebd., S. 24. 
führte auch dazu, dass das Papsttum und folglich die Papstgesandten von dieser Entwicklung betroffen waren. ${ }^{18}$ Wie Just festhält, handelte es sich für die Nuntien um „Zeiten des Umbruchs“, und so sei „der Einfluss des Papsttums“ „in den europäischen Angelegenheiten“ gesunken. ${ }^{19}$ In diesem Spannungsfeld mussten also die Nuntien agieren und „entfalteten wohl noch an den Höfen eine rege diplomatische Tätigkeit zum Schutze der kirchlichen Interessen“. ${ }^{20}$ Doch so einfach war es für die Nuntien - und für den Papst - dann doch nicht, selbst wenn es sich um katholische Fürsten handelte. Die Papstgesandten konnten nicht „auf deren guten Willen“ vertrauen, vor allem wenn die Nuntien auf die katholischen Fürstenhöfe angewiesen waren. Da versagten sich die Fürstenhöfe „immer mehr den päpstlichen Wünschen“. Stattdessen folgten sie den Grundsätzen der Staatsräson, und das konnte auch wirtschaftlicher, militärischer oder geopolitischer Natur sein.

Für die Diplomatie der Päpste bedeutete dies, ihre Strategie so auszurichten, dass durch komplexe Beziehungsstrukturen ihre Ziele und Zwecke umgesetzt werden konnte. Dies geschah, wie Surchat erläutert, „durch ein kompliziertes Netz kleiner politischer Schachzüge“, ${ }^{21}$ und so mussten sie sich auf „die geringen Möglichkeiten“ zurückgreifen, „die sich hier und da“ anboten. ${ }^{22}$ Diese allgemeine Feststellung im 17. Jahrhundert galt auch für den Papstgesandten in Luzern.

Die erste Reform der Nuntiaturen, die in der ersten Hälfe des 16. Jahrhunderts durchgeführt wurden, bestand darin, dass die päpstliche Kanzlei als ausführende Behörde und an deren Spitze der „Secretario intimo“23 sich um die Ernennungen kümmerte. Es war Innozenz VIII. ${ }^{24}$ (1484-1492), der diesen Posten einrichten ließ.25

Unter dem Pontifikat von Papst Gregor XIII. (1572-1585) wurden die zuvor ungeordneten Gesandtschaftsstrukturen neu geregelt. So wurden unterschiedliche diplomatische Rangklassen eingeführt. Ziel war es, die Effizienz zu steigern und damit das Papsttum auf internationaler Ebene zu festigen. ${ }^{26}$

18 Vgl. Leo Just: Die Erforschung der päpstlichen Nuntiaturen, S. 247-248.

19 Zit. nach: ebd., S. 247-248.

20 Vgl. Pierre Louis Surchat: Die Nuntiatur von Ranuccio Scotti in Luzern 1630-1639, S. 24.

21 Zit. nach: Vgl. Pierre Louis Surchat: Die Nuntiatur von Ranuccio Scotti in Luzern 16301639, S. 24.

22 Zit. nach: Leo Just: Die Erforschung der päpstlichen Nuntiaturen, S. 247-248.

23 Er war der Leiter der Geschäfte der päpstlichen Angelegenheiten und Vertrauter des Papstes.

24 Vgl. Marco Pellegrini: Innocenzo VIII, papa.

25 Vgl. Anton Pieper Zur Entstehungsgeschichte der ständigen Nuntiaturen, S. 4.

26 Vgl. Alexander G. Flierl: Diplomatenrecht als Soft Power des Heiligen Stuhls, S. 61. 
In der Hierarchie der Gesandten nahmen die Nuntien die Spitzenposition ein. Ende des 16. Jahrhunderts wurden sie in die aus päpstlicher Sicht wichtigsten Ortschaften entsandt und sollten vor allem als ständige Gesandte auch eine gewisse Kontinuität garantieren. ${ }^{27}$

Dass 1586 Gregors Nachfolger Sixtus V. eine ständige Nuntiatur in der Eidgenossenschaft einsetzte, zeigt, dass es in Rom ein Anliegen war, die Eidgenossenschaft auf dieselbe Bedeutungsebene $\mathrm{zu}$ stellen wie große und wichtige Herrschaftshäuser in Europa. Der damalige Papst hätte auch einen sogenannten Internuntius einsetzen können, der in der Gesandtschaftshierarchie eine zweitrangige Rolle spielte. Auch die Nachfolger von Sixtus V. hielten an der ständigen Nuntiatur in der Eidgenossenschaft fest, womit sich zeigt, dass der Schweiz eine besondere Bedeutung zugemessen wurde. ${ }^{28}$

Bis 1654 waren die Nuntien in der Eidgenossenschaft Diözesanbischöfe. Wie Fink in seinem Werk schreibt, handelt es sich hierbei um die Zeit der „Reformnuntiatur in Luzern“. ${ }^{29}$ Die ständige Nuntiatur in Luzern war somit ein Novum in doppelter Hinsicht. Einerseits war sie durch die Reform der päpstlichen Diplomatie zu einer ständigen Nuntiatur eingesetzt worden, und andererseits war sie als Einrichtung der Reform - also der Anliegen des Konzils von Trient ein neues Mittel, um in das kirchliche Leben in der Eidgenossenschaft wirken zu können.

Die sogenannte „Luzerner Nuntiatur“ war zuständig für die Kantone der Eidgenossenschaft und deren Untertanengebiete, die Drei Bünde (einschließlich Veltlin, Bormio und Chiavenna), das Wallis sowie die gesamten Gebiete der Diözesen Basel, Chur, Konstanz, Lausanne und Sitten, folglich auch das Oberelsass und süddeutsche Gebiete sowie Teile Vorarlbergs und Tirols. ${ }^{30}$ Nach dem Westfälischen Frieden lockerten sich die Beziehungen zu den außerschweizerischen Gebieten zusehends. Ab 1803 war der Nuntius auch bei den konfessionell gemischten, ab 1816 auch bei den reformierten Kantonen akkreditiert. Die Nuntiatur umfasst seither nur noch die Schweiz im heutigen politischen Sinne. ${ }^{31}$

27 Vgl. Pierre Blet: Histoire de la Représentation Diplomatique du Saint Siège.

28 Vgl. Matteo Cantori: La diplomazia pontificia.

29 Vgl. Urban Fink: Die Luzerner Nuntiatur 1586-1873, S. 37-42.

30 Fink ist auch auf das „Problembistum“ Konstanz eingegangen. Vgl. Urban Fink: Der Griff über den Gotthard, S. 113: „Die Krise des Bistums Konstanz war im Gegensatz zu den Schwierigkeiten, mit denen die Bischöfe von Chur, Sitten und Lausanne zu kämpfen hatten, keineswegs eine Folge der Reformation, sondern im wahrsten Sinne „hausgemacht“, ermöglicht durch kirchliche Karrieremuster, die über das Konzil von Trient hinaus und auch gegen dieses Konzil vom Papst und der Kurie zugestanden oder sogar geschützt wurden.“

31 Vgl. Urban Fink: Die Luzerner Nuntiatur 1586-1873. 
Einerseits ging es darum, die protestantischen Kantone $\mathrm{zu}$ „beobachten“, andererseits mussten die Nuntien auch die katholischen Kantone im Auge halten. ${ }^{32}$

In der ersten Phase wurden gewöhnlich Diözesanbischöfe in die Schweiz gesandt, die ihre Bischofssitze beibehielten. Ab 1654 bekleideten die Nuntien den Rang von Titularbischöfen, im 19. Jahrhundert waren es immer mehr Berufsdiplomaten, die den Heiligen Stuhl in der Eidgenossenschaft vertraten. ${ }^{33}$ Rund die Hälfte der Nuntien stammte aus dem Hochadel oder aus in Rom sehr einflussreichen Familien, im 19. Jahrhundert weisen sie bürgerliche Züge auf. Ihre Ausbildung war vor allem auf das Studium der beiden Rechte ausgerichtet, Theologie wurde jedoch nur oberflächlich studiert. Auf theologische Fragen gingen sie in ihren Berichten kaum ein. Ziel der Nuntiaturlaufbahn bildete das Kardinalat. Von 33 Nuntien in der Schweiz im Rang eines Titularbischofs errangen 28 den Kardinalshut, einer - Michelangelo Conti - bestieg 1721 als Innozenz XIII. den päpstlichen Stuhl. ${ }^{34}$

\subsection{Der Umgang mit den Luzerner Behörden}

Luzern $^{35}$ im 16. und 17. Jahrhundert war eine kleine Stadt, die aus italienischer Sicht als „Provinznest“ galt. Es erschien den Nuntien aber, abgesehen von den kulturellen und geographischen Unterschieden, dennoch als vorteilhaft, in dieser Ortschaft zu residieren. Luzern war reisetechnisch einfach zu erreichen, und von dort aus konnte der Nuntius die Ortschaften aufsuchen, die zu seinem Amtsterritorium gehörten.

Der Hauptort der Katholiken in der Schweiz galt seit dem 16. Jahrhundert als „Stadt der Soldaten“. ${ }^{36}$ So stellt Thali fest, dass Luzern „im 16. Jahrhundert weitgehend vom Kriegsdienst für fremde Herren“ lebte. ${ }^{37}$ Wie Kardinal Borromä-

32 Vgl. Eduard His: Die Nuntiatur in der Schweiz.

33 Die Nuntien waren nicht Diplomaten im Sinne, wie es sich erst mit dem Begriff „Diplomatie“ im 18. Jahrhundert etabliert hat. Als „Diplomaten“ waren die Nuntien Ende des 16. Jahrhunderts und dann im 17. Jahrhundert als Akteure die aktiv waren beim Repräsentieren, Austausch und politischen Verhandlungen, die im Namen eines politischen Gebildes - in diesem Falle des Apostolischen Stuhls - mit der Eidgenossenschaft durchführten. Vgl. Stéphane Péquignot: Europäische Diplomatie im Spätmittelalter.

34 Vgl. Jürgen Dendorfer, Ralf Lützelschwab (Hrsg.): Geschichte des Kardinalats im Mittelalter.

35 Vgl. Valentin Groebner: Retroland. Der Historiker geht unter anderem auf die städtebauliche Entwicklung Luzerns ein.

36 Vgl. Johanna Thali: Inszenierung in Text und Bild, S. 540.

37 Zit. nach: ebd., S. 540. 
us in seinen Beschreibungen feststellte, waren die Zentralschweizer - und dazu zählt auch Luzern - durchaus sehr fromme Katholiken. Eine Bestätigung kam auch von den Jesuiten. So beschrieb Jesuitenpater Georg Rotarius in einem Brief von 1575 an den Ordensgeneral folgendermaßen:

Diese Leute sind sehr fromm und sehr eifrig in der göttlichen Kultausübung und sie besuchen oft die Kirchen [...], wo sie sich lange aufhalten. Es gibt Witwen in großer Zahl, denn viele ihrer Ehemänner waren Soldaten, die im Krieg gefallen sind. Deshalb verbringen diese Witwen viel Zeit in den Friedhöfen, um die Gräber ihrer Männer zu pflegen und mit Weihwasser zu segnen. Die Witwen sind ihren Männern treu und verbringen sechs Stunden täglich in den Kirchen für das Gebet. Sie fliehen auch nicht vor der Pest oder anderen ansteckenden Krankheiten [...], obwohl in diesem Ort sehr viel Ehebruch begangen wird, die von Ehefrauen begangen werden, deren Männer im Krieg als Soldaten tätig sind, deshalb müssen wir mit Sorgfalt mit diesen Frauen und Witwen sprechen. ${ }^{38}$

Politisch war Luzern wie die anderen Zentralschweizer Kantone durch die Führung von einzelnen Familien gekennzeichnet. Luzern war ein Stadtstaat, der von Räten regiert wurde. Diese nahmen auch eine Machtstellung gegenüber den ihr zugehörigen Landgebieten und über die Bevölkerung ein. ${ }^{39}$ Dies führte dazu, dass das tägliche Leben sehr reglementiert und kontrolliert wurde. Der Wille zur „Ordnung und Kontrolle“ war von der Auseinandersetzung mit der Reformation geprägt. Um das „Katholisch-Sein“ zu verteidigen, wurden deshalb gezielt Erlasse zum Trink- und Essverhalten sowie zu Kleidung oder Tanzen durchgesetzt. Dazu zählte auch das kirchliche Leben, das in jener Zeit „zunehmend unter die obrigkeitliche Einflussnahme“ geriet. $^{40}$

Bevor die ständige Nuntiatur in Luzern eingeführt wurde, war es der Bischof von Konstanz, der mit Unterstützung der staatlichen Machthaber Luzerns Visitationen des Klerus anordnete und durchführen ließ. Es ging darum, das damals weit verbreitete Konkubinat unter den Priestern zu unterbinden. Eine dazu gehörende Maßnahme war auch die um 1574 beschlossene Berufung der Jesuiten nach Luzern. Dort gründeten sie als Orden, der vor allem im Bildungsbereich tätig war, ein Collegium. Kurz darauf, und zwar 1579, wurde auch die erste päpstliche Nuntiatur eingeführt, die dann ab 1586 zu einer ständigen Nuntiatur wurde. Dazu kam noch die Berufung der Kapuziner 1583, die sich um die „Evan-

38 Eigene Übersetzung aus dem Lateinischen. Zit. nach: Joseph Studhalter: Die Jesuiten in Luzern 1574-1652, S. $358 \mathrm{f}$.

39 Vgl. Johanna Thali: Inszenierung in Text und Bild, S. 540f: „Seit den Anfängen der Reformation und den anschließenden konfessionellen Auseinandersetzungen innerhalb der Eidgenossenschaft zeichnet sich hier eine Politik der Zentralisierung und Intensivierung der Herrschaft ab, die mit einem umfassenden gesellschaftlichen Disziplinierungsprozess einhergeht.“ 40 Zit. nach: ebd., S. 541. 
gelisierung“ der Menschen in jenen Gebieten kümmerten. Man muss also diese drei Maßnahmenpakete zusammen betrachten, um zu sehen, dass es darum ging, nicht nur die Verbreitung der Reformation zu unterbinden, sondern auch eine Stärkung der „eigenen katholischen Identität“ $z u$ fördern. ${ }^{41}$

Eine weitere Besonderheit, die Thali über die Stadt Luzern im 16. Jahrhundert beschreibt, betrifft die „lebendige Spieltradition“. Hier trafen die Nuntien auf eine Gesellschaft, die zwar strengen Regeln unterworfen war und gezwungen wurde, den damaligen katholischen Moralvorstellungen $\mathrm{zu}$ entsprechen, doch trafen sie nicht einfach Menschen, die die ganze Zeit im stillen Kämmerlein oder in den Kirchbänken beteten. Die Luzerner Theatertradition konnte sich durchaus mit den italienischen „compagnie delle commedie“ vergleichen lassen. ${ }^{42}$

Luzern war als Ort bei den Nuntien keineswegs beliebt. Paravicini langweilte sich in Luzern und wollte der Schweiz so rasch wie möglich den Rücken kehren. Bufalini ${ }^{43}$ sprach von der Schweiz als einem „paese disgraziato“, Oddi ${ }^{44}$ bezeichnete Luzern als gottverlassen, und Valenti Gonzaga ${ }^{45}$ sprach vom ungeliebten Luzern als einer „ingrata residenza“, ja sogar von einem „doloroso esilio“. Eine Ursache für das förmliche Unbehagen war das für Südländer ungewohnt nasskalte Wetter. Da Luzern eine kleine Provinzstadt war, fehlte hier ein höfisches Leben, wie es für kirchliche Führungspositionen selbstverständlich war. Die Schweizer wurden als grob, anmaßend, geldgierig und bestechlich bezeichnet. Schmiergelder seien in der Schweiz unerlässlich, schrieb Bonomi 1580 nach Rom. Der Umgang mit den Schweizern sei schwierig, weil sie nicht mit rationalen Argumenten zu überzeugen seien.

Das Organigramm gibt Klarheit über die Organisation der Luzerner Nuntiatur mit ihren drei Bereichen Gericht, Kanzlei und Haushaltung.

Fast 300 Jahre lang beherbergte die Stadt Luzern die bei den katholischen Kantonen der Eidgenossenschaft akkreditierte Apostolische Nuntiatur. Ihr Jurisdiktionsbereich reichte allerdings weit über diesen engeren Bereich hinaus in die süddeutschen Gebiete des Bistums Konstanz, ins Elsass, ins Wallis und in das Gebiet der Drei Bünde bis ins Veltlin hinunter.

Trotz ihrer großen Bedeutung für die Geschichte der katholischen Schweiz befindet sich die wissenschaftliche Erforschung und Aufarbeitung noch in den

41 Vgl. ebd., S. 541: „Diese Institutionen spielen eine wichtige Rolle bei der Durchsetzung der tridentinischen Reformvorschriften, die den politischen Zielsetzungen der Obrigkeit weitgehend entsprechen.“

42 Vgl. Heidy Greco-Kaufmann: Spiegel dess vberflusses vnd missbruchs, S. 63-98.

43 Johannes Octavio Bufalini war von 1755 bis 1759 Nuntius in der Schweiz.

44 Nicolaus Oddi war von 1759 bis 1764 Nuntius in der Schweiz.

45 Luigi Valenti Gonzaga war von 1764 bis 1773 Nuntius in der Schweiz. 
Anfängen. Vor Jahren schon äußerte der damalige Kirchenhistoriker an der Theologischen Fakultät in Luzern, Konstantin Maier, die Erforschung der Luzerner Nuntiatur sei bis heute ein Desiderat. Anfang des 20. Jahrhunderts begannen die Freiburger Professoren Heinrich Reinhardt und Franz Steffens mit der Erforschung der Nuntiatur von Giovanni Francesco Bonhomini (1579-1581), also der Vorphase. Einige Jahre später veröffentlichte Karl Fry zwei Bände zur Nuntiatur von Giovanni Antonio Volpe (1560-1564). Sonst finden sich nur einige kleinere Studien zur Nuntiaturgeschichte. Erst in den letzten Jahren hat das Interesse an dieser Institution wieder zugenommen. ${ }^{46}$

Es ist insgesamt eine Besonderheit, dass gerade die Eidgenossenschaft eine Ständige Nuntiatur erhielt. Im Gegensatz zu anderen Beispielen aus Nordeuropa besteht die Besonderheit auch darin, dass im „Schweizer Fall“ kein Reziprozitätsgedanke im Spiel war, da die Eidgenossenschaft keinen Botschafter in Rom hatte. ${ }^{47}$ Einzig die Bedeutung der Schweizergarde könnte diesbezüglich als eine Art „Schweiz-Vertreter“ beim Papst verstanden werden, doch gerade die „militärische“ Funktion der Nuntien in Luzern, die sich sowohl um die militärisch-diplomatischen Dinge als auch um das Söldnerwesen kümmerten, zeigt die enge Verbindung zwischen päpstlicher Diplomatie und Schweizer Militarismus. ${ }^{48}$

\subsection{Der Umgang mit den eidgenössischen Behörden}

Es war 1586 neben derselben Äußerung Kardinal Borromeos dann auch der Wunsch der Fünf Orte Uri, Schwyz, Ob- und Nidwalden, Luzern und Zug, eine ständige Nuntiatur in Luzern einzusetzen. Die Stadt am Vierwaldstättersee war der sogenannte katholische Vorort, sozusagen die Hauptstadt der „katholischen Schweiz“. Es ist also wichtig festzuhalten, dass es der Wunsch der katholischen Schweizer war, einen solchen Gesandten zu haben, und nicht als „Aufdrängen“ von Seiten des Papstes empfunden wurde, wenn man auch davon ausgehen kann, dass durchaus großes Interesse in Rom bestand, eine direkte Kontrolle über die Katholiken in der Eidgenossenschaft $\mathrm{zu}$ haben und $\mathrm{zu}$ beobachten, was die Protestanten in jener Region vorhatten. ${ }^{49}$

46 Vgl. Urban Fink: Die Luzerner Nuntiatur 1586-1873.

47 Die Errichtung der Luzerner Nuntiatur 1586 steht allerdings im Zusammenhang mit der Errichtung ständiger Nuntiaturen in Graz 1580, Köln 1584 und Brüssel 1596.

$48 \mathrm{Zu}$ Beginn des 16. Jahrhunderts galt die Eidgenossenschaft als eine militärische Macht, die sich in den europäischen Kampf um die Herrschaft über Norditalien eingeschaltet und wichtige Gebiete unter ihre Kontrolle gebracht hatte. Die Expansionsträume endeten im Jahr 1515 vor den Toren Mailands, in Marignano.

49 Vgl. Johann von Müller: Geschichten Schweizerischer Eidgenossenschaft. 
In Luzern blieb die Nuntiatur mit einigen Unterbrechungen bis 1873 bestehen. Sie war somit nach der Botschaft Frankreichs - die in Solothurn residierte - die zweitälteste ständige Gesandtschaft in der Schweiz. ${ }^{50}$

Korrekterweise muss man aber festhalten, dass die Eidgenossenschaft durch ihr politisches und vor allem konfessionell komplexes Gebilde eine Besonderheit war und ihre Komplexität deshalb andere Voraussetzungen als andernorts üblich mit sich brachte. „Bei den meisten Begebenheiten wird die hier angedeutete Entwicklung sichtbar“ ${ }^{51}$ schreibt Surchat dazu.

Während Surchat seine Studie einer einzelnen Nuntiatur - und zwar jene von Ranuccio Scotti - widmet, so hält er fest, dass damit „ein Beitrag zur Geschichte der päpstlichen Politik jener Zeit, aber auch zur langsamen, sich damals anbahnenden Entwicklung zum modernen Staat hin“52 liefern kann. Was in Luzern galt, ihre Krisen und Erfolge, sei auch für „die Kölner, mit der sie wegen der Vielfalt der ihr unterstellten Territorien einiges gemeinsam hatte“, oder „selbst wie die Pariser, von der sie sich schon rein an Bedeutung stark unterschied“, 53 ähnlich, lautet das Fazit von Surchat.

Die Nuntiatur in der Schweiz war seit ihrem Beginn 1586 nicht nur ein administrativer Posten der katholischen Kirche. Vielmehr steckte hinter der Aufgabe des Nuntius auch eine wichtige „kirchenpolitische“ Funktion: Der Nuntius d. h. der Vatikan-Botschafter - beobachtete und mischte sich in das politische Tagesgeschäft der Eidgenossenschaft ein. Diese Botschafter waren - mit einer Ausnahme - immer Italiener, die Karriere an der Kurie machen wollten. Dabei war die Nominierung als Nuntius in der Schweiz ein Zwischenschritt. Die Nuntiatur in der Schweiz, die in der katholischen Stadt Luzern ansässig war, galt nicht unbedingt als irrelevante Stelle, aber auch nicht als letzte Karrierestufe. ${ }^{54}$

Die größten Hürden für die Nuntien bestanden in zweierlei Hinsicht: Einerseits mussten sie in einem „Provinznest“ wie Luzern wohnen und sich andererseits mit komplizierten Problemen auseinandersetzen. Denn die Nuntiatur wurde im Zuge der Gegenreformation durch Kardinal Karl Borromäus eingesetzt. Das Zusammenleben der Katholiken und Protestanten war dementsprechend schwierig. ${ }^{55}$

Man kann zwei Phasen aufzählen: zunächst die der Einsetzung der Nuntiatur von 1586 bis etwa 1604. Es folgte dann ab 1605 bis etwa 1712 die Phase der

50 Vgl. Michael F. Feldkamp: Die europäischen Nuntiaturen in der frühen Neuzeit.

51 Zit. nach: Vgl. Pierre Louis Surchat: Die Nuntiatur von Ranuccio Scotti in Luzern 16301639, S. 24.

52 Zit. nach: ebd., S. 24.

53 Zit. nach: ebd., S. 24.

54 Vgl. Volker Reinhardt: Nuntien und Nationalcharakter.

55 Ebd. 
Konsolidierung. Galt es in der ersten Phase, das Zusammenspiel zwischen dem Nuntius, den führenden katholischen Familien in der Schweiz und der römischen Kurie überhaupt in Einklang zu bringen, folgte in der zweiten Phase der Konsolidierung der Versuch, Kapital aus diesem Zusammenspiel zu schlagen. So gilt als einer der größten Erfolge der Nuntiatur die Gründung der Helvetischen Benediktinerkongregation von $1602 .{ }^{56}$ Ein Jahr später folgte die Schwäbische Benediktinerkongregation. Diese Institutionalisierung diente dem Zweck, den Erhalt und die Reform der Klöster zu sichern. Damit verbunden waren auch die Ziele des tridentinischen Reformwerkes weiter umzusetzen. Was die Nuntien in der Zeit von 1586 bis 1654 vor allem auszeichnete, war ihr Reformeifer. Dieser erlahmte ab der Mitte des 17. Jahrhunderts. Das lag daran, dass sich das Umfeld änderte und somit die Nuntien schwierige Hindernisse überwinden mussten, die die Gesandten bis Mitte des 17. Jahrhunderts nicht hatten. ${ }^{57}$

Allgemein galt im 16. und 17. Jahrhundert ein Grundkonsens zwischen den katholischen Orten, insbesondere Luzern, mit der Nuntiatur. Diese Übereinstimmung ging im Rahmen des Zweiten Villmerger Krieges 1712 verloren. ${ }^{58}$ Zudem setzten im 18. Jahrhundert verstärkt Tendenzen der weltlichen Mächte ein, nichtstaatliche Einflüsse auf ihre Untertanen einzuschränken. ${ }^{59}$

So ging es bei den Streitigkeiten ${ }^{60}$ mit den weltlichen Mächten um Steuerund Gerichtsemtionen sowie um Klöster- und Klerikerbesteuerung. ${ }^{61}$ Mit dem Einfall der Franzosen, dem Zusammenbruch der Alten Eidgenossenschaft 1798

56 Vgl. Charles Benziger: Die diplomatischen Beziehungen des Heiligen Stuhls mit der Eidgenossenschaft.

57 Vgl. Urban Fink: Die Luzerner Nuntiatur 1586-1873. Es begann die Zeit der Positionskämpfe zwischen Kirche und Staat, die das Bild des gesamten 18. Jahrhunderts prägen sollten. Die Nuntien waren immer stärker damit beschäftigt, die klerikalen Vorrechte zu verteidigen. Daher musste es zu massiven Konflikten mit dem sich modernisierenden Staat kommen.

$58 \mathrm{Vgl}$. Volker Reinhardt: Geschichte der Schweiz. Das lag auch daran, dass verschiedene Konflikte zwischen der Gesamtheit der katholischen Schweizer Kantone oder einzelnen Orten mit der Nuntiatur dazu führte, dass sich die Katholiken und die Nuntiatur nicht mehr denselben Konsens teilten. Bei den Streitigkeiten und Meinungsverschiedenheiten handelte es sich um inhaltlich nicht relevante Themen und Bereiche, die jedoch von den Beteiligten oft als wichtig eingestuft wurden. Diese Reibereien zerstörten das bisher gewahrte gute Einvernehmen $\mathrm{zu}$ wesentlichen Teilen.

59 Im Grunde kann man sagen, dass sich der Machtkonflikt zwischen kirchlichen Machtträgern und weltlichen Machthabern spätestens ab Mitte des 18. Jahrhunderts verstärkt wurde. Bereits im 16. und dann auch im 17. Jahrhunderts war dieser Konflikt präsent, jedoch durch die überwiegend starke Positionierung der katholischen Kirche und ihrer Würdenträger eindeutig zu ihren Gunsten ausgerichtet.

60 Ebd. Fink erläutert das Beispiel von 1762, als die Gründung der Helvetischen Gesellschaft in Schinznach den Argwohn des Nuntius erregte, da er ein Überhandnehmen des Indifferentismus befürchtete. 
und der Flucht des Nuntius Pietro Gravina nach Süddeutschland ging die Nuntiatur unter, wie Fink festhält. ${ }^{62}$

Ab 1803 war der Nuntius auch bei den konfessionell gemischten, ${ }^{63}$ ab 1816 auch bei den reformierten Kantonen akkreditiert. Die Nuntiatur umfasst seither nur noch die Schweiz. ${ }^{64}$

\subsection{Gründung der Nuntiaturstelle in Luzern: die Rolle von Kardinal Karl Borromäus}

Die Nuntiatur in der Eidgenossenschaft zählt zu einer der ältesten Einrichtungen dieser Art. Das liegt wohl daran, dass sie aus besonderen „politisch-religiösen“ Gründen entstanden ist. Die ständige Nuntiatur wurde 1586 in Luzern eingerichtet und verdankt dies vor allem Karl Borromäus, der durch seine „Schweiz-Kenntnisse“ den Papst davon überzeugt hatte, eine solche Institution ins Leben zu rufen. Eine erste Hauptfunktion des Nuntius in Luzern war es also, die Entwicklung der Reformation in der Eidgenossenschaft zu beobachten und allenfalls zu reagieren. Die Nuntiatur galt somit lange Zeit als „Vorposten zu den reformiert-protestantischen" Gebieten. ${ }^{65}$

Das Reformkonzil von Trient (1545-1563) bildet die Grundlage der modernen Nuntiaturen. Die Erneuerung der katholischen Kirche nahm mit der Gründung der sogenannten Reformnuntiaturen sichtbare Gestalt an. Aus der Sicht der italienischen Reformkreise besaß die Eidgenossenschaft eine entscheidende

61 Ebd. Ein Beispiel aus der zweiten Hälfte des 18. Jahrhunderts war der Einsatz der Nuntiatur gegen die Druckerei Agnelli in Lugano, die ein Hauptzentrum im Kampf gegen die Gesellschaft Jesu war. Dem Versuch, die Druckerei stillzulegen, kam ein hoher Symbolwert zu. Letztlich war es jedoch ein Kampf gegen Windmühlen.

62 Ebd.

63 Ebd. „1798 wurde der Nuntius von den Franzosen und der Helvetischen Regierung ausgewiesen. 1803 erfolgte die Wiedererrichtung der Nuntiatur. Nach 1815 war die Diözesanregelung eines der Hauptgeschäfte, ab 1830 belasteten die Spannungen zwischen der Kirche und einzelnen liberalen Kantonen (Klosteraufhebungen) die Beziehungen stark.“

64 Ebd. „Im neuen Bundesstaat ließ sich der Heilige Stuhl ab 1848 nur noch durch Geschäftsträger vertreten, die vom übrigen diplomatischen Korps isoliert waren. Der Aufforderung der Gesandten Frankreichs und Österreichs, die Nuntiatur solle nach Bern übersiedeln, kam der Geschäftsträger 1864 nicht nach, weil dies seiner Ansicht nach eine Anerkennung der neuen Bundesverfassung bedeutet hätte. Die Verurteilung des Kulturkampfs in der Schweiz durch Pius IX. führte am 12. Dezember 1873 zum Abbruch der ohnehin getrübten diplomatischen Beziehungen und zur Aufhebung der Luzerner Nuntiatur. Am 12. Februar 1874 verließ der Geschäftsträger die Schweiz.“

65 Vgl. Michael F. Feldkamp: Die europäischen Nuntiaturen in der frühen Neuzeit. 
Schlüsselstellung, um das Eindringen reformatorischer Ideen nach Italien $\mathrm{zu}$ verhindern. Luzern, die wichtigste Stadt der sieben katholisch gebliebenen Orte, bot sich als Sitz des päpstlichen Gesandten an. Nach dem Tode von Karl Borromäus 1584 drängte sich gebieterisch die Stabilisierung der bereits eingeleiteten Maßnahmen auf. Die Errichtung der Luzerner Nuntiatur 1586 steht im Zusammenhang mit der Errichtung ständiger Nuntiaturen in Graz 1580, Köln 1584 und Brüssel 1596.66

Nachdem Karl Borromäus dafür plädiert hatte, eine ständige Nuntiatur einzurichten, wurden die ersten Nuntien nach Luzern geschickt. Die Instruktionen - also „Bedienungsanleitungen“ für die Anwärter - waren vor allem auf jenen Beschreibungen begründet, die Borromäus verfasst hatte. ${ }^{67}$

\subsection{Das Verhältnis der Nuntien zu den Schweizer Bischöfen}

Die Nuntiatur ist eine Bezeichnung, die vor allem das päpstliche Gesandtschaftswesen (Diplomatie) betrifft. Da sie im Laufe des 16. Jahrhunderts in Europa entstanden ist, war anfangs das Verhältnis zu den Ortsbischöfen nicht immer einfach und von Missverständnissen und Kompetenzansprüchen gekennzeichnet. Hinzu kam die Tatsache, dass der päpstliche Gesandte den Rang eines Bischofs einnahm. Der Nuntius übte somit eine Doppelfunktion aus. Einerseits war er als diplomatischer Vertreter des Heiligen Stuhls ein Informationsbeschaffer der römischen Kurie, indem er Informationen zu seinem Gastland sammelte und gleichzeitig von Rom Instruktionen erhielt. Andererseits vermittelte er in seiner kirchlichen Funktion zwischen Bischöfen, Klerus und Kurie und war somit wie ein „Zwischenbischof“. Eine weitere wichtige Aufgabe - und das barg wohl das größte Konfliktpotential - war die Überprüfungsfunktion des Nuntius bei Bischofswahlen und -ernennungen, um die Tauglichkeit der Kandidaten $\mathrm{zu}$ untersuchen. ${ }^{68}$

Waren die zu Beginn des 16. Jahrhunderts in die Eidgenossenschaft entsandten Nuntien vor allem mit politischen Aufgaben wie Militärkapitulationen und der Anwerbung von Söldnern betraut, was das Verhältnis zu den Ortsbischöfen wenig tangierte, wurden die Nuntien nach dem Konzil von Trient vor

66 Vgl. Anton Pieper Zur Entstehungsgeschichte der ständigen Nuntiaturen.

67 Vgl. Stichwort „Karl Borromäus“, in: https://hls-dhs-dss.ch/de/articles/010211/2012-06-25/ (31. Dezember 2020): „Um die Ausbildung und Disziplin des Klerus zu verbessern und gleichzeitig der Verbreitung des Protestantismus Einhalt zu gebieten, regte Borromäus 1579 die Errichtung einer ständigen Nuntiatur in der Schweiz an; da die röm. Kurie aber zögerte, wurde diesem Begehren erst 1586 entsprochen.“

68 Vgl. Alexander G. Flierl: Diplomatenrecht als Soft Power des Heiligen Stuhls. 
allem in den Dienst der Gegenreformation und der Katholischen Reform gestellt. Und seither übten sie zum Teil quasibischöfliche Funktionen aus. Das bedeutet, dass, nachdem 1586 auf Wunsch der Fünf Orte (Uri, Schwyz, Unterwalden, Luzern und Zug) in Luzern, dem katholischen Vorort, ${ }^{69}$ die ständige Nuntiatur errichtet wurde, die mit Unterbrechungen bis 1873 dort ansässig blieb, die römische Kurie einen Gesandten hatte, der die Bischöfe in der Schweiz kontrollieren konnte. ${ }^{70}$

Das Verhältnis zwischen den Nuntien und den Ortsbischöfen in der Eidgenossenschaft war dadurch erschwert, dass es durch parallele Amtshandlungen zu Reibereien kommen konnte. Ein Beispiel dafür konnten die Pontifikalhandlungen durch den Nuntius sein. Dennoch gab es „keinen Zündstoff für eine grundsätzliche Auseinandersetzung “. ${ }^{71}$

Eine Besonderheit dieser diplomatischen Beziehung war der Umstand, dass die Eidgenossenschaft beim Heiligen Stuhl keine ständige diplomatische Mission errichtet hatte. ${ }^{72}$ Nach der französischen Botschaft in Solothurn war sie wie bereits schon oben beschrieben - die zweitälteste ständige Gesandtschaft in der Schweiz. ${ }^{73}$

Die Nuntien hatte es mit sechs Bischöfen zu tun. Am einfachsten war es mit dem Bischof von Como, der für die Tessiner Gebiete zuständig war. Bereits unter Kardinal Borromäus, der Erzbischof von Mailand war, gehörte das Tessin zu jenen Gebieten, die aus römischer Sicht vorbildlich waren.

Größere Schwierigkeiten im 16. und 17. Jahrhundert machten die Bistümer Konstanz und Basel. Die Reformation führte dazu, dass der Bischofssitz von Basel verlorenging und ab 1528 nach Pruntrut übertragen wurde. ${ }^{74}$ Hier lag das Augenmerk der Nuntien im 16. und 17. Jahrhundert vor allem darauf, die Stellung des Bischofs von Basel im Schweizer politischen Kontext zu stärken.

Das Bistum Konstanz bildete den flächenmäßig größten Teil der Nuntiatur. Dort lag das Konfliktpotential vor allem darin, dass der Bischof von Konstanz sich wenig um die Schweizer Gebiete kümmerte und sich die meisten Konstan-

69 Wegen Differenzen mit dem katholischen Vorort residierte die Nuntiatur 1725-30 in Altdorf und 1835-43 in Schwyz. Vgl. Stichwort „Nuntiatur“, in: https://hls-dhs-dss.ch/de/articles/ 011742/2011-11-03/ (31. Dezember 2020).

70 Vgl. Martin Papenheim: Machen Päpste Politik?; Alexander G. Flierl: Diplomatenrecht als Soft Power des Heiligen Stuhls.

71 Zit. nach: Bettina Braun: Princeps et episcopus, S. 191.

72 Erst ab 1991 ließ sie sich durch den Botschafter in Prag in Sondermission vertreten. Er wurde 2004 zum regulären Botschafter ernannt. Vgl. Stichwort „Nuntiatur“, in: https://hlsdhs-dss.ch/de/articles/011742/2011-11-03/ (31. Dezember 2020).

73 Vgl. Urban Fink: Die Luzerner Nuntiatur 1586-1873.

74 Vgl. Stichwort „Bistümer/Diözesen“, in: https://hls-dhs-dss.ch/de/articles/027048/201010-01/ (31. Dezember 2020). 
zer Bischöfe nicht auf derselben Augenhöhe wie die Nuntien betrachteten, da die Fürstbischöfe von Konstanz zu den geistlichen Reichsfürsten zählten. ${ }^{75}$

Die Nuntiatur in der Schweiz umfasste neben den katholischen Kantonen der Eidgenossenschaft, die zum Bistum Basel oder Konstanz gehörten, auch deren Untertanengebiete sowie die Drei Bünde (einschließlich Veltlin, Bormio und Chiavenna) und das Wallis. Chur (Graubünden) und Sitten (Wallis) waren zwei Bistümer, die im 16. und 17. Jahrhundert ebenfalls wie Basel mit Machtansprüchen der weltlichen Machthaber zu kämpfen hatten. In Chur war das Problem ähnlich wie in Basel der Konflikt mit den Herrscherfamilien, der dann im Laufe der Reformation zur konfessionellen Auseinandersetzung führte. ${ }^{76}$ In Sitten hingegen bestand die Konfrontation darin, dass der Bischof mit der Bevölkerung und deren herrschenden Familien über Machtansprüche stritt. In beiden Fällen diente der Nuntius als Vermittler bzw. Impulsgeber, um die Stellung der beiden Bischöfe im weltlich-politischen Bereich zu stärken. ${ }^{77}$

Die Nuntien waren auch für das Oberelsass, einige süddeutsche Gebiete sowie Teile Vorarlbergs und Tirols zuständig, aber da spielte das Verhältnis zu jenen Bischöfen eine marginale Rolle.

Was die Westschweiz betraf, führte die Reformation auch zum Verlust der Bischofssitze von Lausanne (ab 1615 in Freiburg) und Genf (ab 1586 in Annecy). In diesen Fällen waren die Nuntien beauftragt, diese Bischofssitze zu rehabilitieren. $^{78}$

Während des Dreißigjährigen Krieges bestand die Aufgabe der Nuntien vor allem in der Förderung der katholischen Streitparteien. Die Schweiz hatte hier-

75 Vgl. Stichwort „Konstanz (Fürstbistum)“, in: https://hls-dhs-dss.ch/de/articles/008561/ 2008-10-28/ (31. Dezember 2020): „Der Fürstbischof von Konstanz gehörte zu den geistlichen Reichsfürsten; eine Verleihung der Regalien ist erstmals 1248 überliefert.“

76 Vgl. Stichwort „Chur (Diözese, Fürstbistum), in: https://hls-dhs-dss.ch/de/articles/011403/ 2020-01-09/ (31. Dezember 2020): „Die Ilanzer Artikel von 1524 und 1526 reduzierten die bischöfliche Herrschaft auf den noch 1514 von Kaiser Maximilian I. als von der Stadt exemt erklärten Hof Chur, das in Tirol gelegene Fürstenburg, die Herrschaft Grossengstingen in Schwaben (1717 Verkauf an das Kloster Zwiefalten) sowie auf einige Reste der früheren weltlichen Herrschaft in Graubünden, so im Münstertal, in Obervaz und in Fürstenau.“

77 Vgl. Stichwort „Sitten (Fürstbistum)“, in: https://hls-dhs-dss.ch/de/articles/008560/201409-25/ (31. Dezember 2020): „Versuche der Bischofsfürsten Walter Supersaxo und Matthäus Schiner, im Rahmen der Neuaufzeichnung des Walliser Gewohnheitsrechts auch ihre Herrschaft in den Landrechten von 1475 und 1514 gesetzlich zu festigen, scheiterten. 1571 gelang zwar die verfassungsmäßige Verankerung; die weltlichen Rechte des Fürstbischofs waren da als Folge der politisch und konfessionell gespaltenen Landschaft nach Schiner, der als Sachwalter von Papst und Kaiser wie keiner nach ihm die reichsfürstliche Stellung verkörpert hatte, aber faktisch bereits zugunsten der Zenden ausgehöhlt.“

78 Vgl. Stichwort „Nuntiatur“, in: https://hls-dhs-dss.ch/de/articles/011742/2011-11-03/ (31. Dezember 2020) 
bei eine militärische und geopolitische Funktion. Deshalb prägte diese Ausgangslage die Verhältnisse der Nuntien zu den Bischöfen der Schweiz. Nach dem Westfälischen Frieden lockerten sich die Beziehungen zu den außerschweizerischen Gebieten zusehends.

Die Nuntien mussten sich nicht nur mit den Protestanten, den katholischen Patriziern und den Bischöfen in der Schweiz auseinandersetzen: nach französischem Vorbild versuchte auch Österreich, Appellationen Vorderösterreichs an die Luzerner Nuntiatur zu unterbinden. Auf diese Weise gerieten die Nuntien immer stärker in das Spannungsfeld mit den Bischöfen hinein. Ein Beispiel hierfür war der Streit mit dem Fürstbischof von Konstanz um die geistliche Gerichtsbarkeit und um die Klosterfreiheit. 